\title{
Novel Hybrid Architecture of Infotainment for Streaming Signals in Vehicular Network
}

Reshma S ( $\nabla$ reshmas191118@gmail.com )

Global Academy of Technology https://orcid.org/0000-0002-7849-0543

Chetana Prakash

BIET: Bapuji Institute of Engineering \&amp; Technology

Mohamed Rafi

UBDT College of Engineering: University BDT College of Engineering

\section{Research Article}

Keywords: Vehicular Adhoc Network, Infotainment System, Road Side Units, Hybrid Ar-chitecture, OnBoard Unit.

Posted Date: March 16th, 2021

DOI: https://doi.org/10.21203/rs.3.rs-273846/v1

License: (9) This work is licensed under a Creative Commons Attribution 4.0 International License. Read Full License 


\section{Abstract}

Infotainment system is meant for offering integrated services of information and entertainment to the driver to offer a better driving experience. However, the infotainment system's present state is more towards entertainment and less towards accessing real-time information propagation for avoiding the occurrences of road fatalities. In this regard, the streaming of multimedia signals would increase communication to enhance driving experiences. The infotainment system is one integral part of vehicular communication for road safety; however, it has received less attention. Existing approaches towards data transmission among vehicles are studied concerning three standard architectures. A hybrid approach is far better than the infrastructure-based and ad-hoc-based approaches. Still, there are open-end loopholes towards hybrid architectures where reliability in the data dissemination process in the presence of dynamic topology of vehicular nodes is not considered. Therefore, the proposed study introduces a novel analytical model where the vehicles' streaming services can be offered by improvising the existing hybrid architecture. With a unique inclusion of link-quality-based attributes towards making a precise decision of the vehicles' mobility, the proposed system formulates error-free links among the communicating vehicles. The simulated study outcome shows that the proposed system offers better streaming performance than existing hybrid schemes.

\section{Introduction}

There is a significant revolution towards road safety systems and their associated services [1]. The idea is basically to control the accidents while driving or supporting the upcoming application to be required by the drivers for betterment in their driving experience [2]. In this regard, the Vehicular Adhoc Network (VANET) is the prime topic under attention, which lets communication among different vehicles [3]. The complete mechanism of communication in VANET is carried out by complying with three different architectures, i.e., i) fixed access point-based architecture, ii) Adhoc-based architecture, and iii) hybridbased architecture [4]. Various works have been carried out to improve the communication system in VANET using different approaches [5]-[10], and the infotainment system is an integral part of the VANET system with less attention. Our prior work [11][12] discusses in this regard, and this paper has presented a specific solution to address a problem associated with communication in dynamic topology. The infotainment system's evolution is meant for enhancing the propagation of information towards contributing road safety systems.

The existing system has been researched mainly towards disseminating information, a textual format connected with signal propagation. However, this is quite impractical concerning the actual demands of the accessibility of the information. Research work carried out towards multimedia data propagation in vehicular nodes, which cater to real-time communication in case of emergency [13]-[15]. Streaming multimedia files offer a much faster way of communication as well as they are comprehensive too. However, this is not so easy when it comes to implementing it in the real world. There are multiple challenges associated in this regards viz. i) streaming the live feeds of video requires an established connection between two vehicular nodes, which are quite hard to achieve owing to their uncertain 
mobility patterns, ii) streaming data via fixed access point will require higher dependency of resources as well as it will be time-consuming as all the data will be streamed via fixed access points iii) streaming through the ad-hoc mode of architecture will demand an effective routing scheme which can control intermittent connection of vehicular node and this is not present till date, and iv) streaming using hybrid architecture will demand a design of quite efficient and faster decision-making technique which may not give better signal quality and possibly degrade the quality of experience of the user. At present, the infotainment system does not have such possibilities. It is assumed that it will not be challenging to build a firmware with the computational model with the proliferation of a cost-effective embedded system. The infotainment system is only required to configure with the transceiver system within the vehicle called the Onboard Unit of OBU, which is already there under the domain of VANET.

Therefore, the manuscript discusses a novel idea that considers a vehicle's practical mobility scenario to offer streaming services. The proposed study implements a simplified analytical model that facilitates the streaming of massive data for vehicular nodes. The contribution of the proposed study is as follows:

- The proposed study develops a model where multiple vehicles are presented in the mobility environment to reduce the impediments towards streaming video.

- The proposed system introduces a novel attribute called a degree of orientation, which assists in decision-making to formulate effective routes based on the direction of the vehicle's mobility.

- The proposed system introduces a mechanism of selecting the relay node to ensure data carriers' availability so that there is always a seamless transmission of data and services among the vehicles.

The proposed system's significant contribution is that it constructs a route based on link quality considering the direction of the vehicles' mobility. This offers better confirmation of the confirmed connection possibilities because the vehicle is moving over a specific path. The organization of the manuscript is as follows: Sect. 2 discusses the existing approaches where literature based on three different architectures is discussed; Sect. 3 discusses the research problem, while Sect. 4 discusses proposed methodology adopted to address the problems in the existing system. Section 5 discusses the system design, which further elaborates the implementation modules involved in the study. Section 6 discusses the result obtained from implementation, while Sect. 7 summarizes the study contribution in conclusion.

\section{Existing Approaches}

This section discusses the different forms of communication mechanism adopted in the vehicular network in the existing system. It has been noticed that the approaches used for mitigating various situations in the vehicular network are of three forms viz. i) infrastructure-based, ii) Adhoc-based, and iii) hybrid-based. The work being carried out in each of these cases is as follows:

\subsection{Infrastructure-based Approach}


In this approach, the vehicular nodes communicate with the infrastructure (Road-Side Unit-RSU) as the fixed access point to carry out communication. The access point offers information about the routing and traffic to the vehicular nodes, which enable one vehicle to communicate with another vehicle. However, it should be noted that this form of solution is quite expensive (Fig. 1). The infrastructure-based approach usually adopts the principle of dissemination routing, further classified into two forms Urban Multihop Broadcast [16] and Optimized Dissemination of Alarm Message [16].

At present, different approaches have been evolved up towards this form of communication. Unique work presented by Huang et al. [17] has discussed an optimal policy towards RSU. The decision-making is formulated based on the distance between the vehicle and RSU considering delay due to queuing. The existing approach also emphasizes distributed infrastructure to facilitate effective data dissemination (Luan et al. [18]). Such a scheme emphasizes replicating content in RSU considering the access delay of contents while optimizing the utility offered by a global network. Studies towards security and communication are also considered, considering the infrastructure-based scheme (Zhang and Boukerche [19]). Challenges associated with reliable data communication between vehicle and infrastructure is addressed in Alsharif and Shen [20]. Zhang et al. [21] have performed a predictive strategy for the performance of connection in both uplink and downlink transmission for the infrastructure. Studies toward the optimal selection of infrastructure are carried out by Silva et al. [22].

\subsection{Adhoc-based Approach}

This communication strategy enables one vehicle to communicate directly with another vehicle without dependency on any access point. Each vehicular node acts as a router and assists in data transmission and exchange between the communicating nodes. The vehicular nodes are also required to cooperate while adopting this form of scheme. The ad-hoc approach is found to have two main classes, i.e., topology-based routing [16] and opportunistic-based routing [16]. Topology-based routing is again found to be of two forms, i.e., Reactive and proactive forms. Some standard implemented communication approach for reactive routing is Adhoc On-demand Distance Vector (AODV), Adhoc On-Demand Multipath Distance Vector (AOMDV), and Destination Sequence Distance Vector (DSDV). In contrast, opportunistic routing is of two forms, MaxProb [16] and Vehicle Assisted Data Delivery (VADD) [16].

Existing literature towards Adhoc based approach has emphasized opportunistic routing schemes. Adoption of game theory for a minimal price of data dissemination has been carried out by Yang et al. [23]. It is also explored that channel scheduling significantly assists in opportunistic routing, as Al-Fuqaha et al. [24]. The work addresses challenges in predicting traffic patterns with a solution that does not demand an apriori information network. A predictive scheme has also been studied by Li et al. [25], where signal quality is being predicted for assessing vehicle utility scores.

Further, the routing performance improvement in such a network can be optimized using a bio-inspired scheme (Tian et al. [26]). This scheme is also known for its adaptive nature facilitating transmission over a dynamic environment of vehicular nodes. Adopting opportunistic routing with the cooperative transmission was also witnessed to offer improved communication in the vehicular Adhoc network (Peng 
et al. [27]). The trajectory-based approach is also found to offer seamless routing for nodes in a dynamic environment for improved data communication using a simplified strategy (Dau and Labiod [28]). Cao et al. [29] carried out a unique implementation, where the idea assists in data propagation, focusing on the vehicle's charging efficiency. The study also showcases that the adoption of inclusion of bus significantly assists in the data dissemination process.

\subsection{Hybrid Approach}

This form of communication strategy integrates both infrastructure and ad-hoc-based approaches. This kind of approach usually makes use of geographic routing, which is broadly classified as Greedy Perimeter Stateless Routing (GPSR) and Geographic Source Routing (GRS) [16].

There have been various studies carried out considering a hybrid approach towards communication. Khan et al. [30] have used fuzzy logic and genetic algorithm for solving optimization issues in faster communication in the vehicular ad-hoc network. Adopting the hybrid approach complements visible light communication, too, with a limited communication range (Chen et al. [31]. Forms of study are more inclined towards infrastructure management and less towards the ad-hoc network. There are also studies related to enhancing hybrid communication (Zhu et al. [32]). Such hybrid schemes show that transmission degradation in the hybrid network can be solved by the integrated usage of a greedy approach for data propagation and recovery system. Another scheme of the hybrid networks uses routing using traffic-based clusters (Qi et al. [33]), which optimizes the data delivery system. Such a scheme is claimed to balance overhead, delay, and cost of channel capacity. A study towards reliable data transmission in the hybrid approach was carried out by Feng et al. [34], which offers lesser dependencies towards traffic density and fixed access points. GSR is another dominant approach in the hybrid scheme. Various researchers (Salkuyeh et al. [35], Lee et al. [36], Hussen et al. [37], O’Driscoll et al. [38]) have used this scheme with different mechanisms to enhance communication. There have been various efforts towards improving GPSR scheme too (Malik et al.[39], Garrosi et al. [40], Bengag et al. [41], Setiabudi et al. [42], Yang et al. [43], Liu et al. [44], etc.).

The next section offers a briefing of problems associated with existing approaches discussed in the literature, followed by the proposed solution.

\section{Research Problem}

A closer look into the existing approaches highlights that they are more inclined towards adopting different conventional variants of routing schemes. Still, they are less focused on catering to innovative application designs. Majority of the upcoming application on modern communication in vehicles demands transmission of the heavier multimedia signals between the vehicles-such forms of application targets for enhancing the experience of onboard users. The existing approach discussed in the prior section does not consider the challenges associated with the transmission of heavier files. The contributor to such a challenge is dynamic topology as well as an increased rate of data transmission. The problems increase double-fold when an increased degree of quality is demanded with the massive 
size of heavier data. Existing approaches using multipath propagation use partitioning of the data content to be forwarded by different routes. Although such schemes reduce data rates' complexity, they do not include variability of node coverage under different routes for vehicular nodes. Therefore such schemes will eventually lead to congestion, collision, and fluctuating contention, which is highly detrimental for quality communication in a vehicular network. Therefore, there is a need to formulate a mechanism considering this above fact of communication.

It has also been seen in existing approaches where a geographic scheme of routing is used to construct a communication path. However, such approaches depend on positional information of a vehicle and secondary attributes, e.g., speed, direction, orientation, etc. Such schemes are not inclusive of the reliability factor out of dynamic topology, which does not offer much concrete evidence of such schemes' sustainability in the long term. The challenging aspect of such a scheme is that they do not consider stability in the load balancing process to forward heavier data files. The biggest challenge in the existing hybrid scheme, which is considered an effective scheme in most scenarios, is that it considers a communication vector presence when the multipath is selected, followed by resisting interference. This is a slightly impractical assumption as it is less likely to occur in the practical world.

To mitigate the heavier data transmission challenge, it is needed to consider various intrinsic and extrinsic attributes connected to the vehicles' dynamicity. Further, there is a need to frame up a computational model that offers i) faster response time, ii) higher quality of signal transmission, iii) costeffective algorithm implementation. From the existing study, it is clear that the hybrid approach is the most suitable approach to address and model this problem; however, the issues associated with both infrastructure and ad-hoc nature in hybrid approach is required to be sorted out. Hence the core research problem will be to use a hybrid approach and develop a reliable communication system among vehicles in a dynamic environment. The next section discusses the proposed solution.

\section{Proposed Methodology}

The proposed solution towards addressing existing problems is to adopt a hybrid vehicular network approach and develop a novel model for effective streaming of data. The proposed system is developed considering a case study of the infotainment system, patched up with the Onboard Unit (OBU) of a vehicle. The infotainment system is assumed to do two tasks i) act as an interface for multimedia streaming-based communication among multiple vehicles, and ii) it supports connectivity with the wireless network (both infrastructure and ad-hoc) on the backend for facilitating the communication system. Figure 4 highlights the proposed scheme.

According to Fig. 4, the proposed system takes data input to be relayed from one vehicle to another to support an upcoming streaming application. The upper block relates to the inclusion of the fixed access point coordinated with the $\mathrm{OBU}$ and external networks (either by using a fixed access point or connected to another vehicle). It manages both data as well as services. The next lower block is about clustering grid, which partitions the complete area of communication and movement of the vehicle into specific 
geometric zones with a predefined area of clusters. Each vehicular node under one cluster formulates a group using an Adhoc-based communication scheme. This group further interacts with the fixed access point if required. When the destination's information is known to the next specific number of neighboring vehicular nodes, the routes are formed in an ad-hoc manner. However, if the routes cannot be established within their time-out period, the system switches over to a fixed access point. The idea is to ensure that the source vehicle could stream the data to the destination node with higher reliability. Hence, a hybrid approach is used in the proposed system to improve the reliability of data transmission. The system introduces a degree of orientation parameter, which offers more decision-making to formulate routes. A further selection of a relay node is carried out to ensure that stream data reach the destination node with a higher success rate of communication.

\section{System Design}

The implementation of the proposed system is carried out considering the practical environment of the vehicular network. To target the processing of a massive stream of data between two communicating mobile nodes, various scenarios must be considered and implemented in developing this framework.

\subsection{Smart OBU for Infotainment System}

$\mathrm{OBU}$ or On-Board Unit is an embedded device that acts as a communication bridge between the vehicle and transceiver (road-side unit) in a vehicular network. The proposed system assumes that the infotainment system is directly connected to the OBU. Resources for both OBU and infotainment are limited concerning memory, processing capability, and operational speed. The only difference is the memory of OBU cannot be extended, while that of the infotainment system can always is extended using an external storage unit. Hence, one of the challenges in this design process is how to optimize the performance of OBU with limited resources. The proposed system considers a finite number of supportable services by the considered infotainment system and a fixed number of inlet/outlet connections. This connectivity port acts as a bridge of internal interaction between the vehicle/user and OBU.

Figure 5 highlights that the $\mathrm{OBU}$ unit is connected to various components, which increasing its processing capability, in fact, indirectly. This makes the OBU unit smarter when it works alongside with infotainment system. Using the infotainment system's extensive resources, a buffer can be constructed to store the incoming queue of the data stream and filter out quality-oriented data. The proposed system can be considered a set of algorithms that are now possible to be executed within these smart OBU units to process a massive data stream.

\subsection{Clusteringgrid}

The proposed system introduces a novel and simplified topology of the vehicular network to facilitate quality data transmission among the vehicular nodes. Consider the following scenario of data transmission, as exhibited in Fig. 6. Assume a segment of a cross-section of a road with three vehicular 
nodes, i.e., $n_{1}, n_{2}$, and $n_{4}$, moving at a variable speed. These vehicular nodes have smart OBU and assume RSU stationed at a uniform distance over the road's edge. Considering a scenario that $n_{1}$ is interested in forwarding a message to $n_{4}$. However, $n_{1}$ is only in the communication range of $n_{2}$ vehicle and not $n_{4}$ vehicle (Fig. 6(a)). Consider a scenario where a third vehicular node $n_{3}$ is approaching towards the range's direction, which connects $n_{2}$ and $n_{4}$ so that $n_{3}$ becomes an intermediate node (Fig. 6(b)). A third scenario where the third vehicular node $n_{3}$ (acting as an intermediate node between $n_{2}$ and $n_{4}$ ) is found to move away. Consider a parameter $\theta$ to represent the degree of orientation over the vehicular cloud representing the affinity of establishing established communication among the communicating vehicular nodes. A closer look over the scenario stated in Fig. 6(b), and Fig. 6(c) will show that the parameter $\theta$ is highly dependent on the quality of signal exchange between the OBU of all the vehicular nodes $\left(n_{1}, n_{2}, n_{3}\right.$, and $n_{4}$ ) that are associated with multihop-based connectivity. It will also mean that maximization of this parameter $\theta$ will also reduce the propagation of artifacts present in the communication process (owing to density, interference, scattering, fading, etc., in wireless vehicular nodes). Therefore, the proposed system emphasizes evaluating this parameter $\theta$ before processing massive streams of data. Hence, the proposed system's topology is formulated in the grid, where clustering is carried out to offer better distinction among the communicating zones.

The proposed system considers the complete area of simulation and classifies them equally in multiple coverage areas. The coverage area is decided based on the presence of RSU, where each area has one RSU and multiple vehicular nodes with smart OBU. This coverage area is termed a cluster maintained in the form of a grid for the uniform division of the complete simulation area. The study also assumes that each cluster executes a local communication protocol followed by vehicles within the respective cluster. It will mean that two different vehicles in two different clusters are assumed to have two different communication protocols. Therefore, this makes the environment more practical and assists in communication. The study also considers that gateway nodes are connected to all RSU to assists in translational services of heterogeneous routing for two vehicular nodes under different clusters. Apart from this, each cluster also keeps track of the parameter $\theta$ for all local active communicating nodes, assisting nodes in other clusters for undertaking decisions while performing massive data streaming. Figure 7 pictorially showcase the connectivity among vehicles, RSU, and gateway node. The proposed system considers a case study of an urban vehicular network where the vehicles are authorized to move on a defined route over the deployment area. It is to be noted that Line-of-Sight (LOS) issues in the wireless transmission are considered in this deployment region.

\subsection{Selection of Relay Node}

The conventional vehicular network is constructed considering the position, speed, and several vehicles as elementary simulation parameters before constructing the routing process. However, the practical scenario slightly differs from this simulated scenario in various aspects. The proposed system hypothesizes that the distance between two vehicular nodes and their movement direction plays an essential role. Hence, the inclusion of these parameters is required to construct a practical scenario of 
implementation. The adoption of this scenario helps the information of a better form of stabilized link in the presence of a challenging mobility context.

Figure 8 highlights the vehicle's movement's pictorial representation in a different direction and its possible effect on establishing the communication link between the source and destination node. The mobility pattern of a vehicle is completely an unpredictable process, and there is a higher probability of having inclusion of various forms of artifacts over the routes of mobility. This artifact could be high if a vehicular cloud is considered in the mobility model. Hence the formulation of a stabilized communication link between the transmitting and vehicular receiver nodes is quite challenging to be established. The proposed model uses the degree of orientation $\theta$ associated with the vehicular node to decide the routes with a higher probability of delivering the data packet. The degree of orientation $\theta$ is based on the highest probability of the data transmission from the first (transmitting node or relay node) to the second node (relay node or destination node). Therefore, the proposed system undertakes multiple test scenarios of mobility with the presence of three types of nodes, i.e., i) transmitting nodes, ii) receiving nodes, and iii) relay nodes. The test scenario also considers all these vehicular nodes traveling over specific routes equivalent to practical world routes while they move in different directions.

i) Test-Scenario-1: In this test scenario, all the vehicular nodes are moving on routes in a similar direction with different coverage capabilities (Fig. 8(a)). This scenario states that the degree of orientation is much favorable for $n_{2}, n_{4}$, and $n_{7}$ vehicular nodes. The relative degree of orientation for this test environment is shown in Table 1. The inference of the expression associated with the selection of routes is as follows: owing to the weak coverage range of $n_{5}$, the neighboring node $n_{2}$ will select $n_{4}$ where the probability of degree of orientation is better compared to $n_{5}$ (although a degree of orientation for $n_{5}$ is maximum at this point.). Similarly, assuming $n_{6}$ is out of the coverage area of $n_{4}, n_{7}$ is the best possibility with the highest degree of orientation. Hence, the selected relay nodes are $n_{2}, n_{4}$, and $n_{7}$.

Table.1 Exploring Route in the first Case of Mobility

\begin{tabular}{|llll|}
\hline $\begin{array}{l}\text { Vehicular } \\
\text { Nodes }\end{array}$ & $\begin{array}{l}\text { Respective Degree of } \\
\text { Orientation }\end{array}$ & $\begin{array}{l}\text { Relative Degree of } \\
\text { Orientation }\end{array}$ & $\begin{array}{l}\text { Selected } \\
\text { Relay }\end{array}$ \\
\hline$n_{1}$ & $\theta_{1}$ & $\theta_{1}>\theta_{2}$ & $\mathrm{n}_{2 \rightarrow} \mathrm{n}_{4 \rightarrow} \mathrm{n}_{7}$ \\
\hline $\mathrm{n}_{2}$ & $\theta_{2}$ & $\theta_{3}>\theta_{4}$ & \\
\hline $\mathrm{n}_{3}$ & $\theta_{3}$ & $\theta_{2}=0$ & \\
\hline $\mathrm{n}_{4}$ & $\theta_{4}$ & $\theta_{7}>\theta_{6}$ & \\
$\mathrm{n}_{5}$ & $\theta_{5}$ & $\theta_{5}=\max$ \\
\hline $\mathrm{n}_{6}$ & $\theta_{6}$ & $\theta_{8}>\theta_{6}$ & \\
\hline$n_{7}$ & $\theta_{7}$ & $\theta_{8}>\theta_{2}$ & \\
\hline
\end{tabular}


ii) Test Scenario-2:In this case (Fig. 8(b)), the environment chooses certain relay vehicular nodes $\left(n_{2}\right.$ and $n_{5}$ ) to be moving in opposite direction excluding transmitting $\left(n_{1}\right)$ and vehicular receiver node $\left(n_{6}\right)$.In this case (Table 2), the favorable relay nodes selected are $n_{2}, n_{5}, n_{4}$, and $n_{7}$. The justification is: vehicular node $n_{2}$ is the most eligible node which carries the data from $n_{1}$ owing to two reasons, e.g., i) $n_{3}$ moves far away from the destination node, and the degree of orientation of $n_{3}$ is 0 and ii) degree of orientation of $n_{2}$ is higher compared to $n_{3}$. The system selects $n_{4}$ via selected $n_{5}$ as the degree of orientation for $n_{4}$ is better than that of $n_{5}$. A closer look at this scenario is that node $n_{4}$ is a very important relay node as it offers the higher assurance of data delivery right from node $n_{2}$ or node $n_{5}$. Hence, in case of failure to receive data from $n_{5}$, it will always have a significant chance to obtain the same from the $n_{2}$ node. Hence, the reversed direction of nodes $n_{2}$ and $n_{5}$ compliments the data delivery process. Similarly, node $n_{7}$ offers the highest degree of orientation in the intermittent link between $n_{4}$ and $n_{6}$.

Table 2

Exploring Route in the second Case of Mobility

\begin{tabular}{|llll|}
\hline $\begin{array}{l}\text { Vehicular } \\
\text { Nodes }\end{array}$ & $\begin{array}{l}\text { Respective Degree of } \\
\text { Orientation }\end{array}$ & $\begin{array}{l}\text { Relative Degree of } \\
\text { Orientation }\end{array}$ & $\begin{array}{l}\text { Selected } \\
\text { Relay }\end{array}$ \\
\hline $\mathrm{n}_{1}$ & $\theta_{1}$ & $\theta_{1}=\max$ & $\mathrm{n}_{2 \rightarrow} \mathrm{n}_{5 \rightarrow}$ \\
\hline $\mathrm{n}_{2}$ & $\theta_{2}$ & $\theta_{4}>\theta_{3}, \theta_{2}=0$ & $\mathrm{n}_{4 \rightarrow} \mathrm{n}_{7}$ \\
\hline $\mathrm{n}_{3}$ & $\theta_{3}$ & $\theta_{2}=0$ & \\
\hline $\mathrm{n}_{4}$ & $\theta_{4}$ & $\theta_{7}>\theta_{6}$ & \\
\hline $\mathrm{n}_{5}$ & $\theta_{5}$ & $\theta_{5}>\theta_{4}$ & \\
\hline $\mathrm{n}_{6}$ & $\theta_{6}$ & $\theta_{8}>\theta_{6}$ & \\
\hline$n_{7}$ & $\theta_{7}$ & $\theta_{8}=\max$ & \\
\hline
\end{tabular}

iii) Test Scenario-3: In this test scenario, the most challenging and practical situation is considered where transmitting node $n_{1}$ and receiving node $n_{6}$ are traveling in the reverse direction (Fig. 5(c)). The scenario also considers certain candidate relay nodes are also reverse to each other in the movement on the specific routes. The selection of the first relay node $n_{2}$ bears a similar condition-based on the degree of orientation, as discussed in the prior two test scenarios. The next possibilities are vehicular relay nodes $n_{5}$ and $n_{4}$. A closer look at the vehicular node $n_{5}$ shows a better probability of orientation than the other relay node $\mathrm{n} 4$, which is traversing in a direction opposite to itself and receiver node $n_{6}$. Based on the relative values of degree of orientation (Table 3), this scenario does not call for selecting $n_{4}$ as a relay node, and hence both $n_{4}$, as well as $n 7$, are not selected as relay node owing to lesser probability to reach receiver node $n_{6}$ on time. Hence, the only probability for a better degree of orientation and reach ability relies on vehicular relay node $n_{5}$. Therefore, this test scenario selects only $n_{2}$ and $n_{5}$ as a relay node. 
Table 3

Exploring Route in the first Case of Mobility

\begin{tabular}{|llll|}
\hline $\begin{array}{l}\text { Vehicular } \\
\text { Nodes }\end{array}$ & $\begin{array}{l}\text { Respective Degree of } \\
\text { Orientation }\end{array}$ & $\begin{array}{l}\text { Relative Degree of } \\
\text { Orientation }\end{array}$ & $\begin{array}{l}\text { Selected } \\
\text { Relay }\end{array}$ \\
\hline$n_{1}$ & $\theta_{1}$ & $\theta_{1}=\max$ & $\mathrm{n}_{2 \rightarrow} \mathrm{n}_{5}$ \\
\hline $\mathrm{n}_{2}$ & $\theta_{2}$ & $\theta_{3}<<\theta_{4}, \theta_{2}=0$ & \\
$\mathrm{n}_{3}$ & $\theta_{3}$ & $\theta_{2}=0$ & \\
\hline $\mathrm{n}_{4}$ & $\theta_{4}$ & $\theta_{7}>>\theta_{6}$ & \\
$\mathrm{n}_{5}$ & $\theta_{5}$ & $\theta_{5}>>\theta_{4}$ \\
$\mathrm{n}_{6}$ & $\theta_{6}$ & $\theta_{6}=\max$ \\
\hline$n_{7}$ & $\theta_{7}$ & $\theta_{8}=\max$ \\
\hline
\end{tabular}

Hence, the degree of orientation not only depends upon the distance, but it also depends upon the probability of higher proximity of the relay nodes to the receiver node for the successful delivery of data packets. Therefore, this process results in the establishment of multiple hops between the transmitting and receiving vehicular nodes. It should also be understood that this process results in the exploration of stabilized routes in vehicular networks' dynamic scenario. After the effective multi-hop routes are explored and confirmed, then the data packets are transmitted. However, the study does not allow any waiting period for routes to be established between the transmitting and receiving node as this will further lead to uncertainty in established route formation. Hence, for faster delivery, the proposed system permits the transmitting node to explore the neighboring relay node and transmit the data packet to it. All the relay nodes further contribute to this process until the data packet reaches the destination node. To support the streaming of signals, the proposed system formulates a multi-hop communication system. To deal with the network resources' restrictive capacity (e.g., bandwidth), the proposed system splits the streamed data-based on several relay nodes being found in the route exploration process. This mechanism ensures faster transmission with higher reliability to forward the data packet towards its destination node.

\section{Result Analysis}

The proposed system's implementation is carried out in MATLAB considering the following simulation parameters: simulation area: $1000 \times 1100 \mathrm{~m} 2$, time of simulation: 300 s, vehicles: 500 , range of transmission: $100 \mathrm{~m}$, packet size: 2000 bytes, and speed of vehicle: $3-10 \mathrm{~m} / \mathrm{s}$. The performance metric considered for the study is signal quality, response time, delay, and throughput. The complete analysis is carried out using 2500 simulation iteration. Each iteration consists of random construction of path and disseminating data packets by the vehicular nodes with an Omni-directional antenna model. The 
outcome has also been compared with the existing hybrid approach viz. GPSR and GSR protocols over similar testbed to arrive at outcomes.

The outcome shown in Fig. 9 infers that the proposed system offers a significantly higher quality of signal compared to the existing system. Signal quality is computed using the peak signal to noise ratio to assess the streaming quality. The prime reason behind better signal quality can be justified with the vehicles' availability on different routes, even in the presence of dynamic topology. This ensures nodes' presence to transmit the data seamlessly; further relay node is another contributor that does not let the signal quality drop.

Figure 10 and Fig. 11 represent response time and delay, where an interesting outcome has arrived. Although the response time is lower than GSR and GPSR, they are not significantly different. The proposed system maintains a lower response time until 2000 rounds while it starts to increase. Increasing simulation rounds will also mean the degradation of the node's lifetime, causing increasing dependency of resources and hence response time shoots up. However, this fact can be compensated with the respective outcome of signal quality, which is higher for the proposed system. Existing approaches include an iterative mechanism to find the routes every time the one transmission is over, which causes increased response time. However, a closer look at delay performance shows a different aspect (Fig. 10). With the greater availability of selected relay nodes, the transmitting node does not need much effort to stream the destination node's data. A further degree of orientation helps maintain the routes' sustainability based on the vehicle's e dynamic mobile. Hence, the proposed system offers better delay performance.

Figure 12 showcases that the proposed system offers significantly better throughput in contrast to the existing system. The mechanism of selecting the relay node is the prime reason behind this seamless transmission causing increasing throughput.

\section{Conclusion}

The present paper has discussed streaming massive data over the vehicular network adhering to the improvised hybrid architecture. The idea of this work mainly orients around accessing critical online services to enhance the driving experience and reduce road fatalities. The proposed system constructs a novel model of infotainment system where multipath information dissemination is carried out. In large and dense vehicle mobility scenarios, various challenges can obstruct the seamless communication process. This is the prime research challenge addressed in current work. The proposed system introduces certain novelty in designing this hybrid approach by including i) selecting relay node and ii) degree of orientation. These two parameters are critically responsible for decision-making to ensure that data, once propagated, should reach the destination node. The simulation study carried out in MATLAB shows that the proposed system offers faster response time, higher throughput, reduced delay, and increased signal quality. 


\section{References}

1. Kim, Y., \& Lee, T.-J., "V2loT Communication Systems for Road Safety," in IEEE Wireless Communications Letters, vol. 8, no. 5, pp. 1460-1463, Oct. 2019.

2. Zhao, H., Yu, D., Li, T., Mao, and Zhu, H. "Vehicle Accident Risk Prediction Based on AdaBoost-SO in VANETs," in IEEE Access, vol. 7, pp. 14549-14557, 2019.

3. Al-Heety, O. S., Zakaria, Z., Ismail, M., Shakir, M. M., Alani, S., and Alsariera, H. "A Comprehensive Survey: Benefits, Services, Recent Works, Challenges, Security, and Use Cases for SDN-VANET," in IEEE Access, vol. 8, pp. 91028-91047, 2020.

4. Alves, J., \& Wille, E. C. G. "Routing in Vehicular Ad Hoc Networks: Main Characteristics and Tendencies," Journal of Computer Networks and Communications, 2018.

5. Mchergui, A., \& Moulahi, T. B.Alaya, and S.Nasri, "A survey and comparative study of QoS aware broadcasting techniques in VANET," Telecommunication Systems, vol. 66, no. 2, pp. 253-281, 2017.

6. Ksouri, C., Jemili, I., M.Mosbah, and A.Belghith, "VANETs Routing Protocols Survey: Classifications, Optimization Methods, and New Trends," International Workshop on Distributed Computing for Emerging Smart Networks, Springer, Cham, pp. 3-22, 2019.

7. Ghebleh, R. (2018). "A comparative classification of information dissemination approaches in vehicular ad hoc networks from distinctive viewpoints: A survey". Computer Networks, 131, pp. 1537.

8. Berger, C. (2013). C, "Bridging Physical and Digital Traffic System Simulations with the Gulliver TestBed. In M. Berbineau et al, (eds) Communication Technologies for Vehicles", Nets4Cars/Nets4Trains. Berlin: Lecture Notes in Computer Science, Springer.. In.

9. Sheikh, M. S., \& Liang, J., "A comprehensive survey on VANET security services in traffic management system," Wireless Communications and Mobile Computing, 2019.

10. Fotros, M., Rezazadeh, J., and O. A.Sianaki. "A Survey on VANETs Routing Protocols for loT Intelligent Transportation Systems." In Workshops of the International Conference on Advanced Information Networking and Applications, Springer, pp. 1097-1115, Cham, 2020.

11. Al-Smadi, A. M., Alsmadi, M. K., Baareh, A. K., Almarashdeh, I., Abouelmagd, H., \& Ahmed, O. S. S. "Emergent situations for smart cities: a survey," International Journal of Electrical \& Computer Engineering, pp. 2088-8708, 9, 2019.

12. Reshma, S., Prakash, C., Rafi, M., Poornima, B., "Framework for Infotainment System for Processing Massive Data Stream of Critical Online Services," International Journal of Innovative Technology and Exploring Engineering (IJITEE), vol.9, issue. 2, 2019.

13. Qiao, J., He, Y., \& Shen, X. S. (2018). Improving video streaming quality in $5 \mathrm{G}$ enabled vehicular networks. IEEE Wireless Communications, 25, no.(2), pp. 133-139.

14. Lai, C.-F., Chang, Y.-C., Chao, H.-C., Hossain, M. S., \& Ghoneim, A. (2017). A buffer-aware QoS streaming approach for SDN-enabled $5 \mathrm{G}$ vehicular networks. IEEE Communications Magazine, 55, no.(8), pp. 68-73. 
15. Qadri, N. N., \& Fleury, M. (2010). M.Altaf, and M.Ghanbari, "Multi-source video streaming in a wireless vehicular ad hoc network". IET Communications, 4, no.(11), pp. 1300-1311.

16. Alves, J., \& Wille, E. C. G. "Routing in Vehicular Ad Hoc Networks: Main Characteristics and Tendencies," Journal of Computer Networks and Communications, 2018.

17. Huang, L., Jiang, H., Zhang, Z., Yan, Z., and Guo, H. (2018). "Efficient Data Traffic Forwarding for Infrastructure-to-Infrastructure Communications in VANETs" in. IEEE Transactions on Intelligent Transportation Systems, 19, no.(3), pp. 839-853., , March.

18. Luan, T. H., Cai, L. X., Chen, J., Shen, X. S., and Bai, F. (2014). Engineering a Distributed Infrastructure for Large-Scale Cost-Effective Content Dissemination over Urban Vehicular Networks" in. IEEE Transactions on Vehicular Technology, 63, no.(3), pp. 1419-1435., ", , March.

19. Zhang, Q., \& Boukerche, A. (2018). "A Novel Infrastructure-Based Worm Spreading Countermeasure for Vehicular Networks" in. IEEE Transactions on Intelligent Transportation Systems, 19, no.(7), pp. 2188-2203., , July.

20. Alsharif, N., \& Shen, X. "\$i\$CAR-II: infrastructure-based Connectivity Aware Routing in Vehicular Networks," in IEEE Transactions on Vehicular Technology, vol. 66, no. 5, pp. 4231-4244, May 2017.

21. Zhang, W., Chen, Y., Yang, Y., Wang, X., Zhang, Yu, Xuemin Hong, and Mao, G. (2012). Multi-hop connectivity probability in infrastructure-based vehicular networks. IEEE Journal on Selected Areas in Communications, 30, no.(4 (), 740-747.. "." ).

22. Silva, C. M., Meira, W., and Sarubbi, J. F. M. (2016). "Non-Intrusive Planning the Roadside Infrastructure for Vehicular Networks" in. IEEE Transactions on Intelligent Transportation Systems, 17, no.(4), pp. 938-947., , April.

23. Yang, F., Yan, J., Guo, Y., and Luo, X. "Stackelberg-Game-Based Mechanism for Opportunistic Data Offloading Using Moving Vehicles," in IEEE Access, vol. 7, pp. 166435-166450, 2019.

24. Al-Fuqaha, A., Gharaibeh, A., Mohammed, I., Hussini, S. J., Khreishah, A., and Khalil, I., "Online Algorithm for Opportunistic Handling of Received Packets in Vehicular Networks," in IEEE Transactions on Intelligent Transportation Systems, vol. 20, no. 1, pp. 285-296, Jan. 2019.

25. Li, N., Martínez-Ortega, J., Díaz, V. H., and Fernandez, J. A. S., "Probability Prediction-Based Reliable and Efficient Opportunistic Routing Algorithm for VANETs," in IEEE/ACM Transactions on Networking, vol. 26, no. 4, pp. 1933-1947, Aug. 2018, doi: 10.1109/TNET.2018.2852220.

26. Tian, D., Zhou, J., Wang, Y., Sheng, Z., Duan, X., and Leung, V. C. M. (2019). Channel access optimization with adaptive congestion pricing for cognitive vehicular networks: an evolutionary game approach. IEEE Transactions on Mobile Computing, 19, no.(4), pp. 803-820.

27. Peng, F., Zhang, G., Huang, X., Ye, X., and Wu, M., "A Novel TDMA-MAC Protocol for VANET Using Cooperative and Opportunistic Transmissions," 2015 IEEE 82nd Vehicular Technology Conference (VTC2015-Fall), Boston, MA, pp. 1-2, 2015.

28. Huy, N. D., \& Labiod, H., "Opportunistic trajectory-based routing for V2V communications," IEEE 22nd International Symposium on Personal, Indoor and Mobile Radio Communications, Toronto, pp. 783$787,2011$. 
29. Cao, Y., \& Wang, N. (2017). "Toward Efficient Electric-Vehicle Charging Using VANET-Based Information Dissemination" in. IEEE Transactions on Vehicular Technology, 66, no.(4), pp. 28862901., , April.

30. Khan, A. A., Abolhasan, M., Ni, W., Lipman, J., and Jamalipour, A. "A Hybrid-Fuzzy Logic Guided Genetic Algorithm (H-FLGA) Approach for Resource Optimization in 5G VANETs," in IEEE Transactions on Vehicular Technology, vol. 68, no. 7, pp. 6964-6974, July 2019.

31. Chen, J., \& Wang, Z. "Topology Control in Hybrid VLC/RF Vehicular Ad-Hoc Network," in IEEE Transactions on Wireless Communications, vol. 19, no. 3, pp. 1965-1976, March 2020.

32. Zhu, L., Li, C., Xia, B., He, Y., and Lin, Q., "A Hybrid Routing Protocol for 3-D Vehicular Ad Hoc Networks," in IEEE Systems Journal, vol. 11, no. 3, pp. 1239-1248, Sept. 2017.

33. Qi, W., Landfeldt, B., Song, Q., Guo, L., and Jamalipour, A. "Traffic Differentiated Clustering Routing in DSRC and C-V2X Hybrid Vehicular Networks," in IEEE Transactions on Vehicular Technology, vol. 69, no. 7, pp. 7723-7734, July 2020.

34. Feng, D., Yajie, M., Fengxing, Z., Xiaomao, W., and Kai, H. "A Safety Message Broadcast Strategy in Hybrid Vehicular Network Environment," The Computer Journal, vol. 61, no. 6, pp. 789-797, June 2017.

35. AsgharpoorSalkuyeh, M., \& Abolhassani, B. (2016). "An Adaptive Multipath Geographic Routing for Video Transmission in Urban VANETs" in. IEEE Transactions on Intelligent Transportation Systems, 17, no.(10), pp. 2822-2831., , Oct.

36. Lee, C., Lo, S., Tang, M., Horng, and Kuo, Y., "A hybrid traffic geographic routing with cooperative traffic information collection scheme in VANET," 13th International Conference on Advanced Communication Technology (ICACT2011), Seoul, 2011, pp. 1496-1501.

37. Hussen, H. R., Choi, S., Kim, J., and Park, J. "Stateless and predictive geographic multicast scheme in flying Ad-hoc networks," 2017 Ninth International Conference on Ubiquitous and Future Networks (ICUFN), Milan, 2017, pp. 685-690.

38. O'Driscoll, A., \& Pesch, D., "Hybrid geo-routing in urban vehicular networks," 2013 IEEE Vehicular Networking Conference, Boston, MA, 2013, pp. 63-70.

39. Malik, R. F., Nurfatih, M. S., Ubaya, H., Zulfahmi, R., and Sodikin, E., "Evaluation of greedy perimeter stateless routing protocol on vehicular ad hoc network in Palembang city," 2017 International Conference on Data and Software Engineering (ICODSE), Palembang, 2017, pp. 1-5.

40. Garrosi, M. T., "Enhanced Intersection-Based Perimeter Geo-Routing in Urban Vehicular Ad-Hoc Networks," 2016 IEEE 84th Vehicular Technology Conference (VTC-Fall), Montreal, QC, 2016, pp. 1-5.

41. Bengagand, A., \& Boukhari, M. E. "Enhancing GPSR routing protocol based on Velocity and Density for real-time urban scenario," 2020 International Conference on Intelligent Systems and Computer Vision (ISCV), Fez, Morocco, 2020, pp. 1-5.

42. Setiabudi, A., Pratiwi, A. A., Ardiansyah, D., Perdana, \& Sari, R. F., "Performance comparison of GPSR and ZRP routing protocols in VANET environment," 2016 IEEE Region 10 Symposium (TENSYMP), Bali, 2016, pp. 42-47. 
43. Yang, X., Li, M., Qian, Z., and Di, T. "Improvement of GPSR Protocol in Vehicular Ad Hoc Network," in IEEE Access, vol. 6, pp. 39515-39524, 2018.

44. Liu, H., Qiu, T., Zhou, X., Chen, C., \& Chen, N. "Parking-Area-Assisted Spider-Web Routing Protocol for Emergency Data in Urban VANET," in IEEE Transactions on Vehicular Technology, 69, no. 1, pp. 971982, Jan. 2020.

Figures

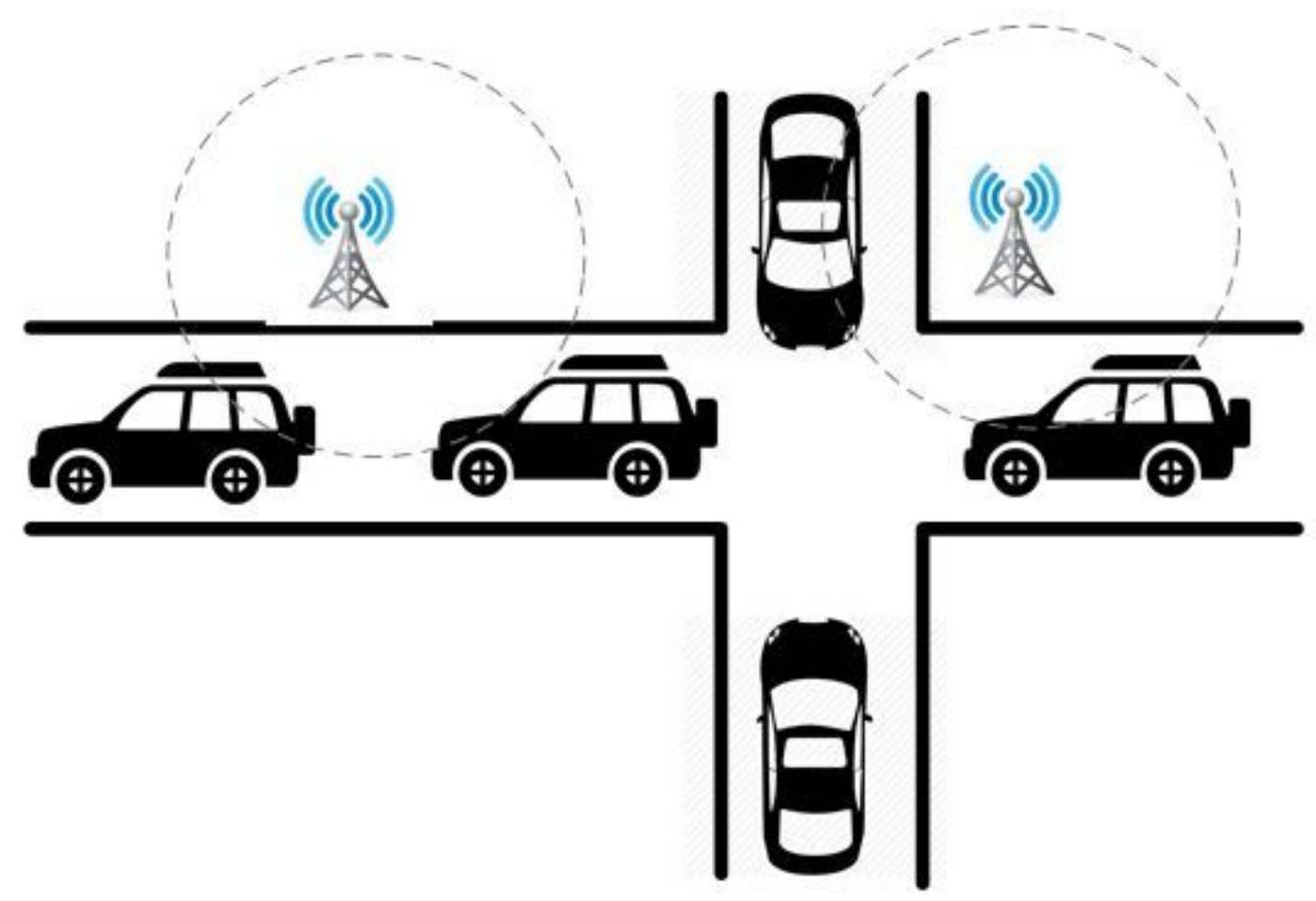

Figure 1

Infrastructure-based approach. Vehicles communicate with fixed access point present over the road (called as Road Side Unit) 


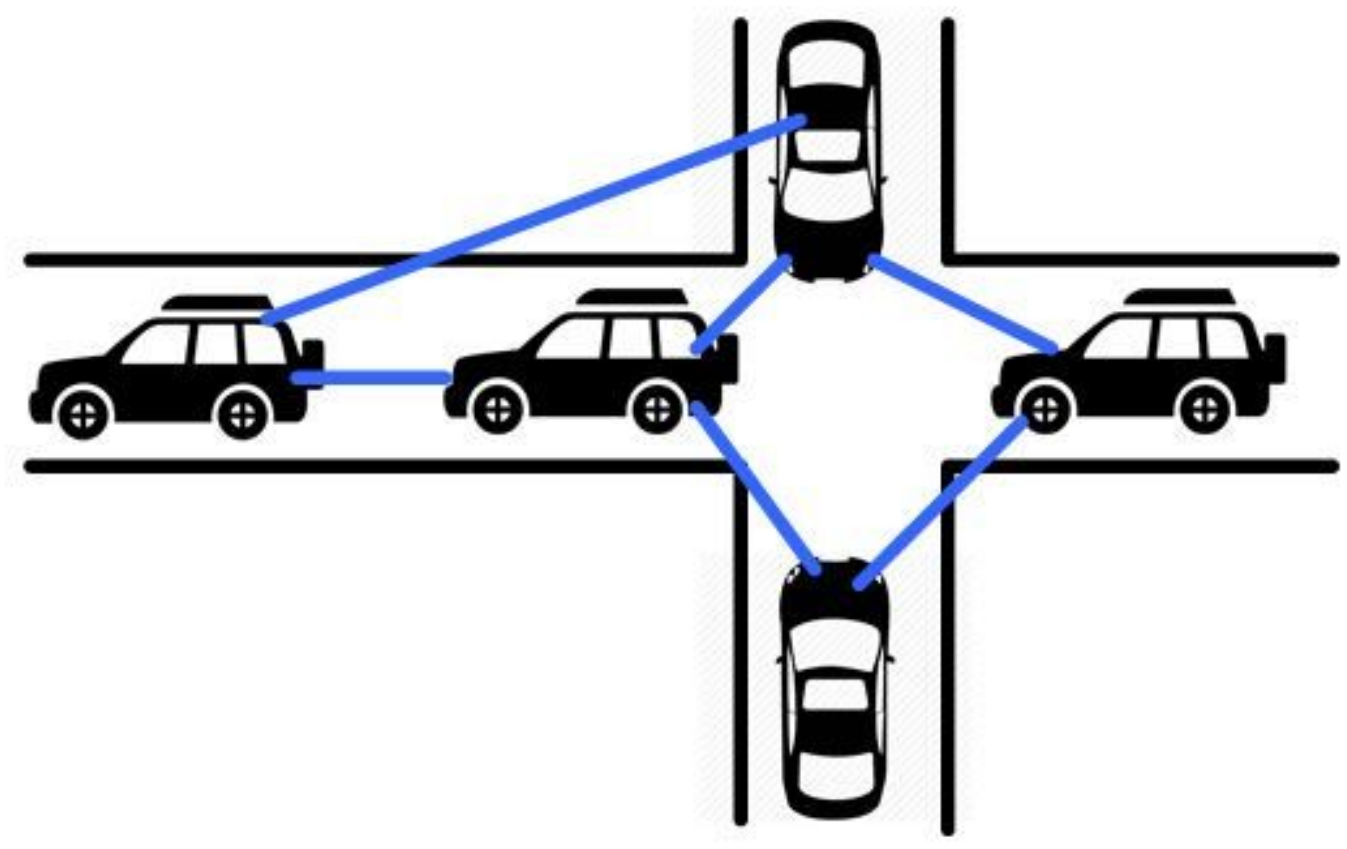

Figure 2

Adhoc Based Approach. Vehicles communicate among themselves, acting as a self-router 


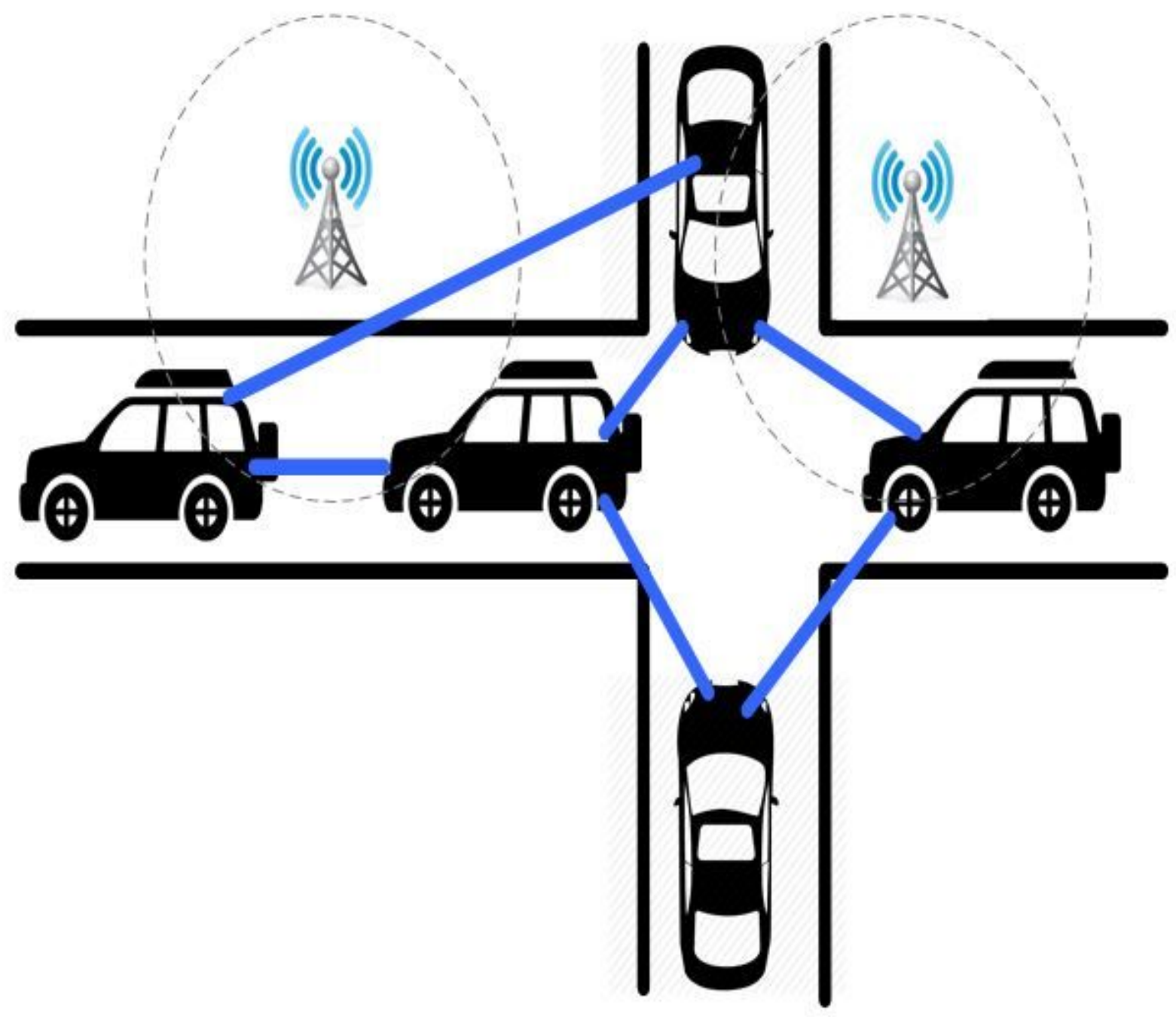

Figure 3

Hybrid based approach. This approach considers an ad-hoc network that connects the presence of a fixed access point, as well as each vehicle 


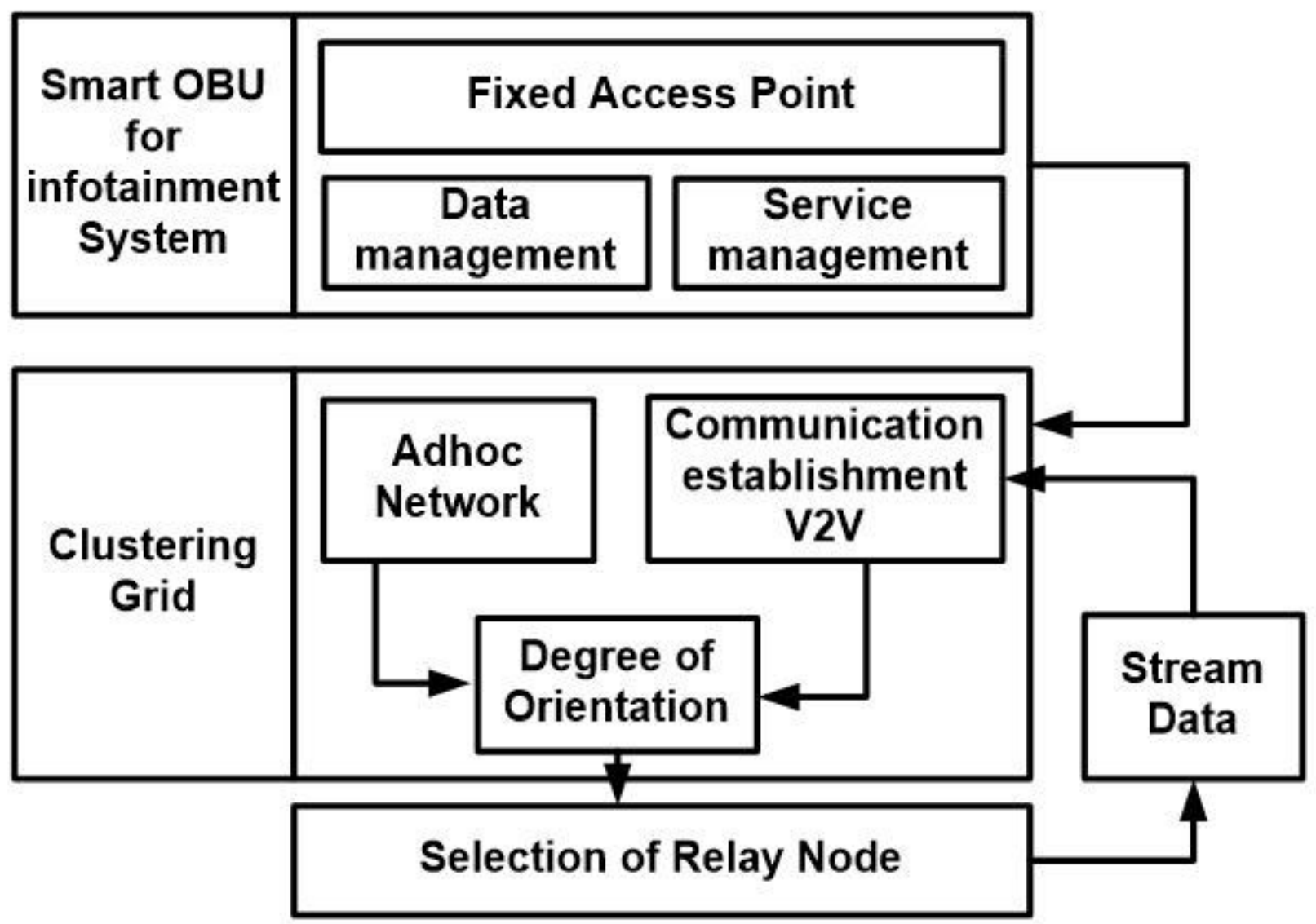

Figure 4

Proposed Scheme of Data Streaming in Hybrid Vehicular network. The scheme consists of three blocks of operation i) Smart OBU for the infotainment system, ii) Clustering Grid, and iii) Selection of Relay Node.

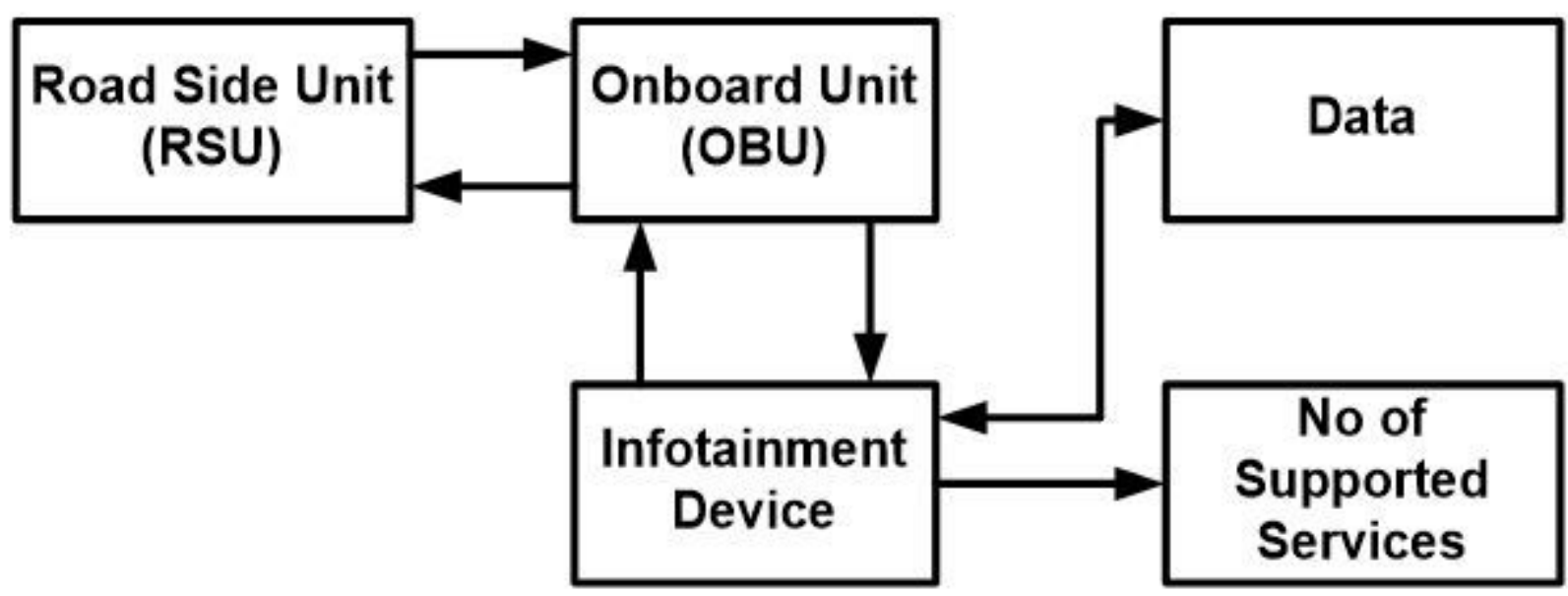

Figure 5 


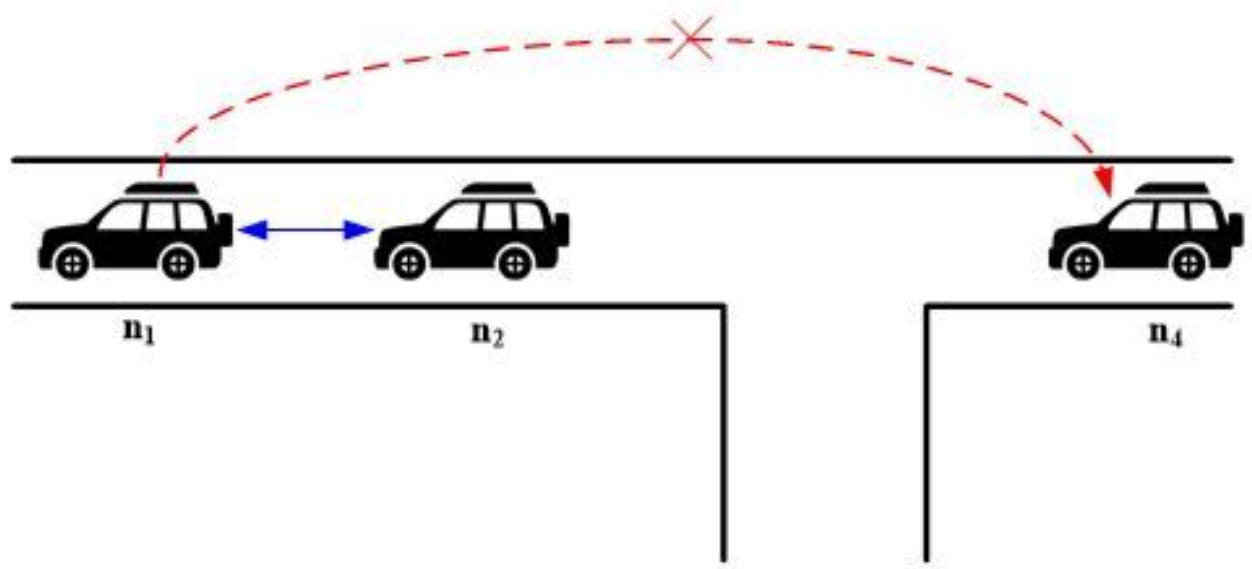

(a)

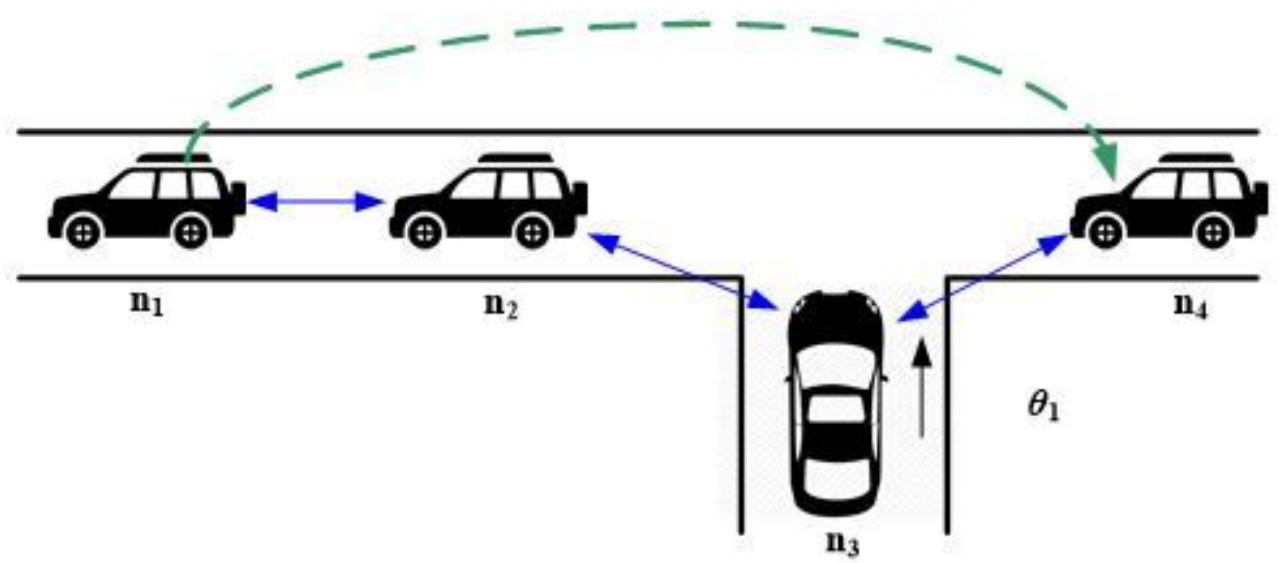

(b)

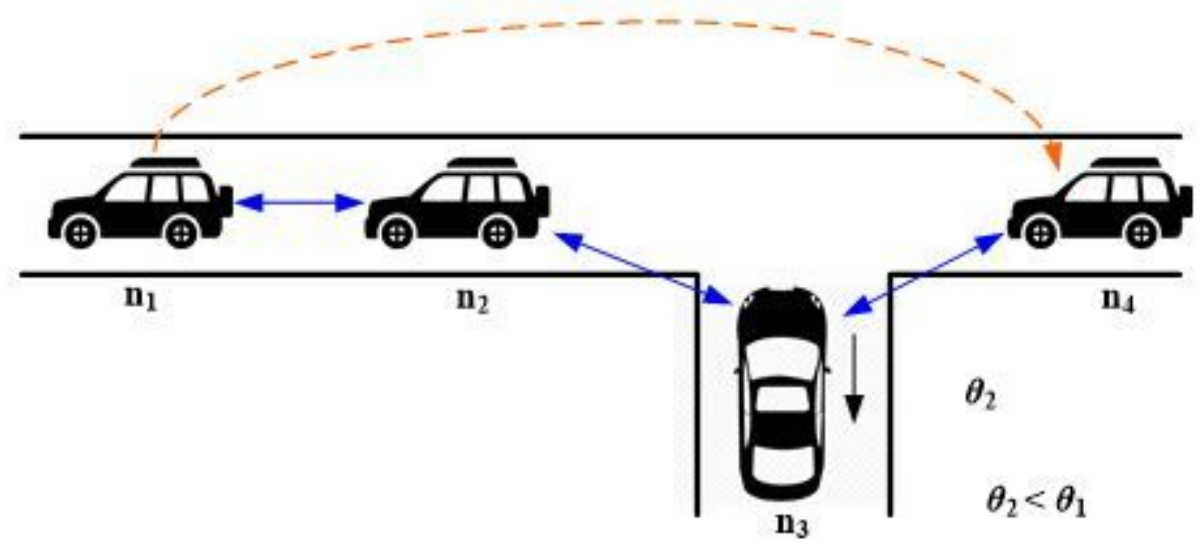

(c)

Figure 6

Consider Possible Scenario of Vehicular node communication. (a) source node $\mathrm{n} 1$ communicates with $\mathrm{n} 2$ but cannot communication with its destination node $n 4$, (b) $n 1$ can now communicate with $n 4$ via intermediate nodes $\mathrm{n} 2$, and $\mathrm{n} 3$ node with a higher degree of orientation, i.e., $\theta 1$ is spontaneously higher, 
(c) node $\mathrm{n} 3$ moves away from both $\mathrm{n} 2$ and $\mathrm{n} 4$ leading to degradation of $\theta 2$, which also weakens the connectivity between $\mathrm{n} 1$ and $\mathrm{n} 4$.

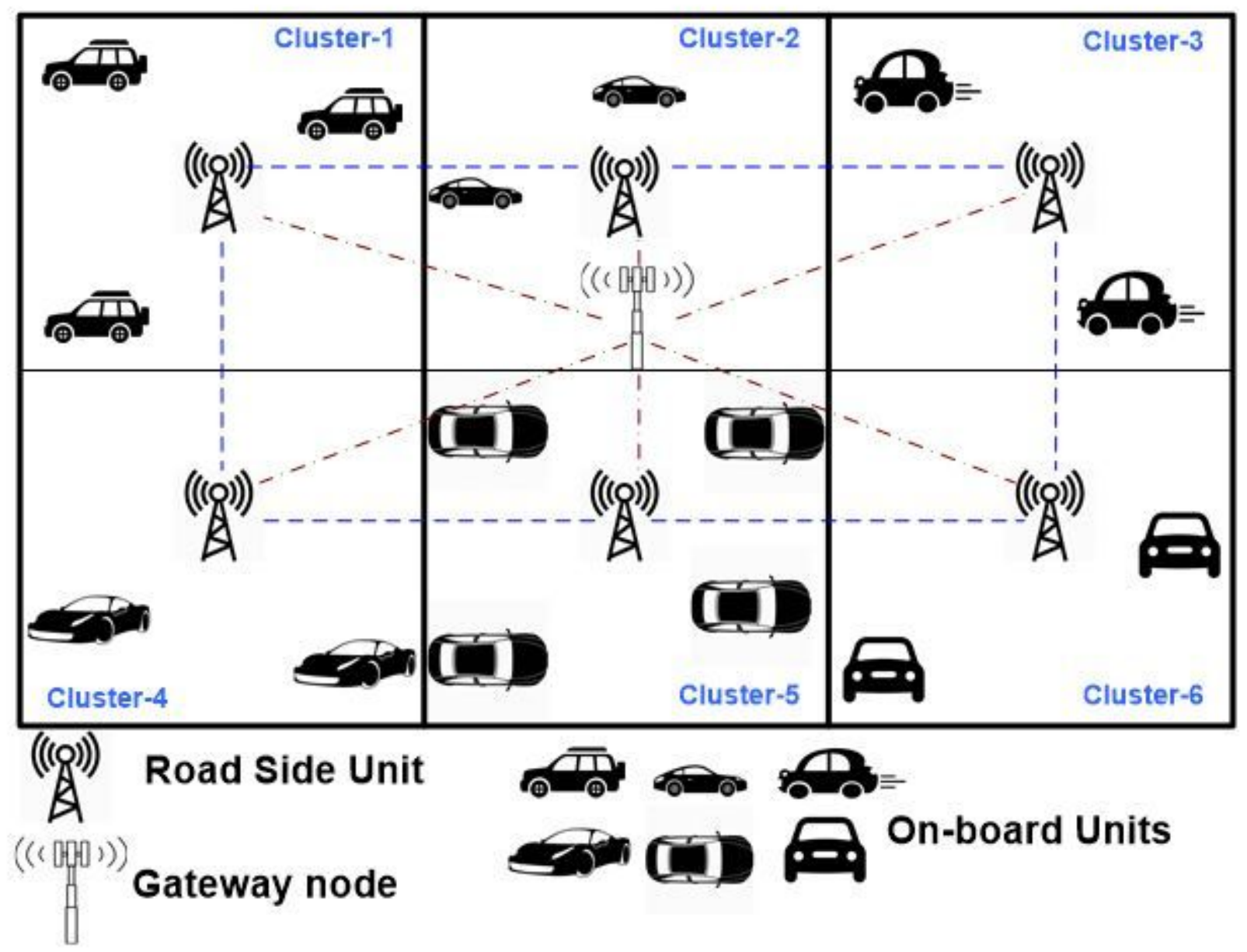

Figure 7

Deployment of the vehicles in the new topology. In a sample of 6 clusters, different vehicles (or OBU) are deployed which are connected to their respective RSU. All the RSU are finally synced with a gateway node for assists in heterogeneous data streaming in a vehicular network. 


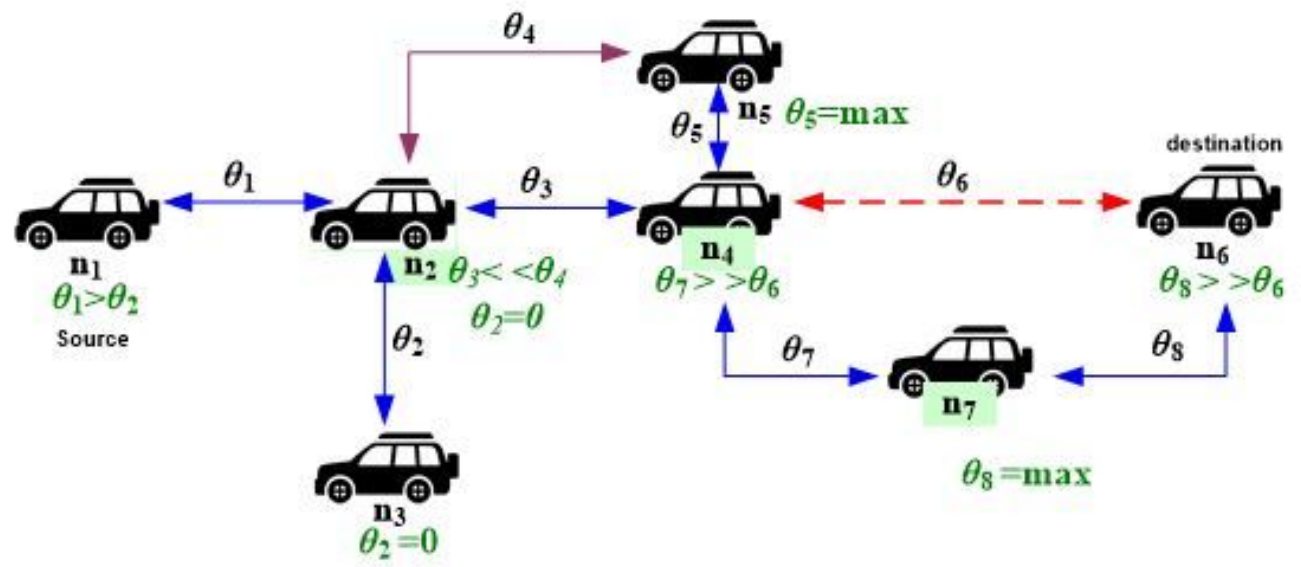

(a)

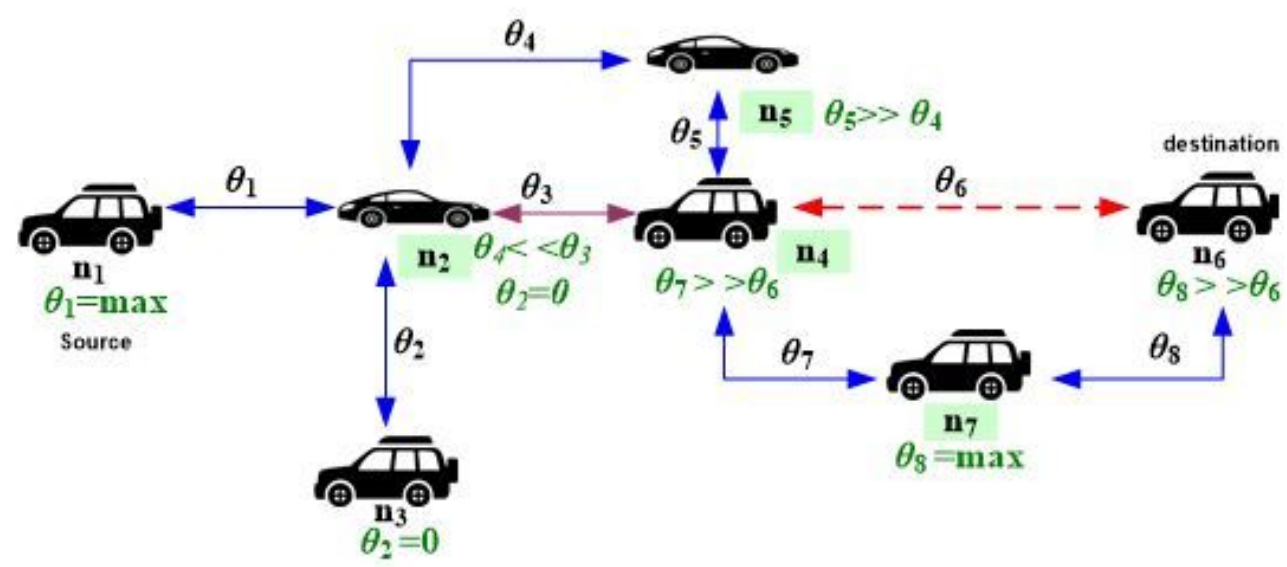

(b)

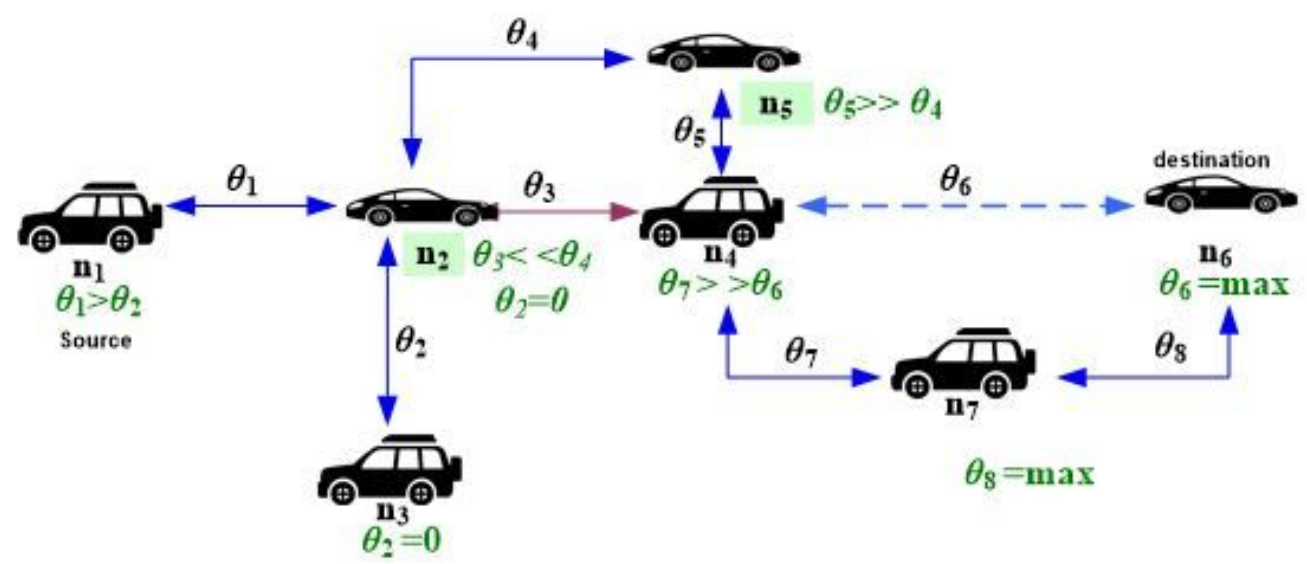

(c)

Figure 8

Process of selecting relay node in three test scenarios viz a) one-directional vehicles, ii) relay nodes in the opposite direction, and iii) destination node is moving at opposite direction along with some specific relay node 


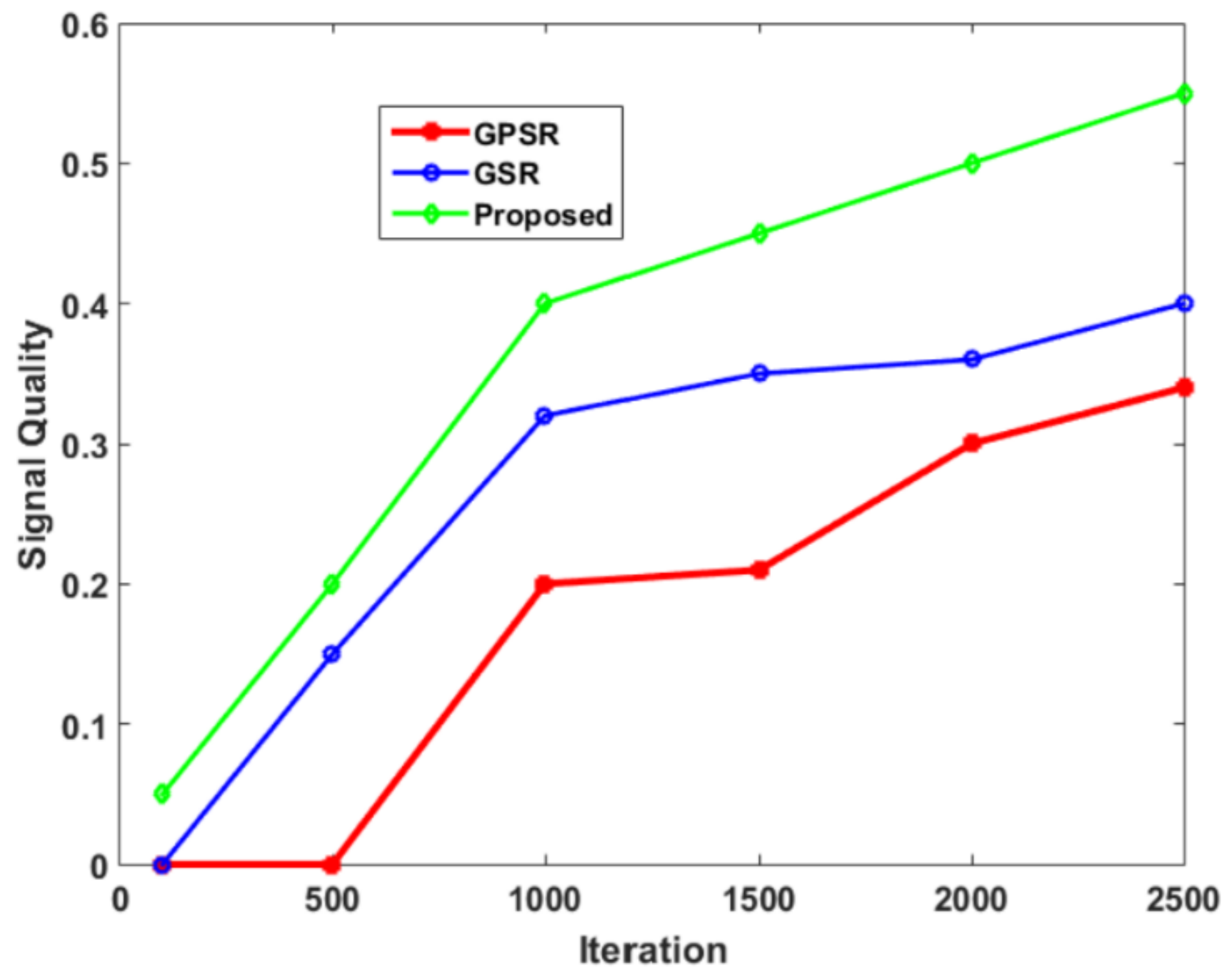

Figure 9

Comparative Analysis of Signal Quality 


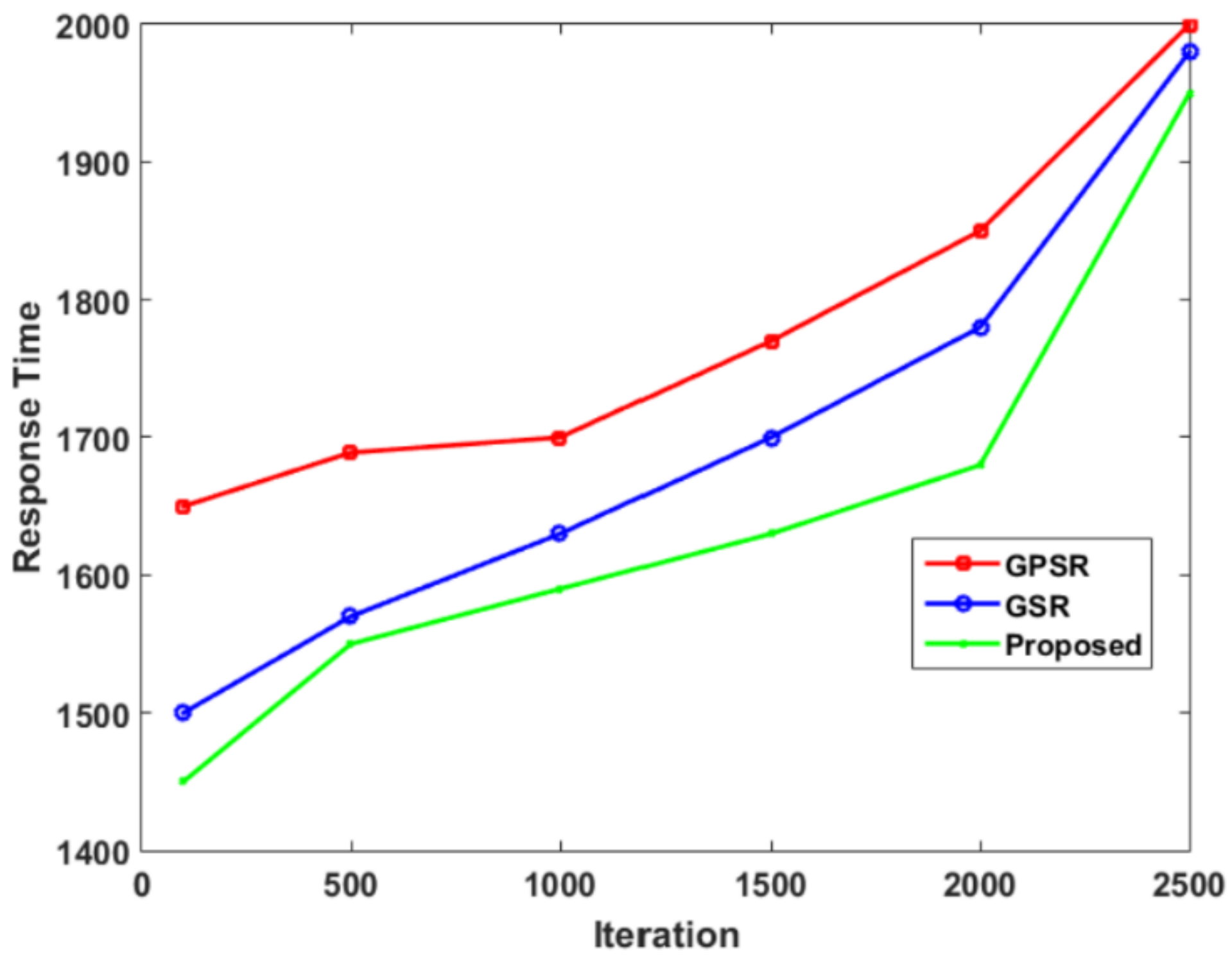

Figure 10

Comparative Analysis of Response Time 


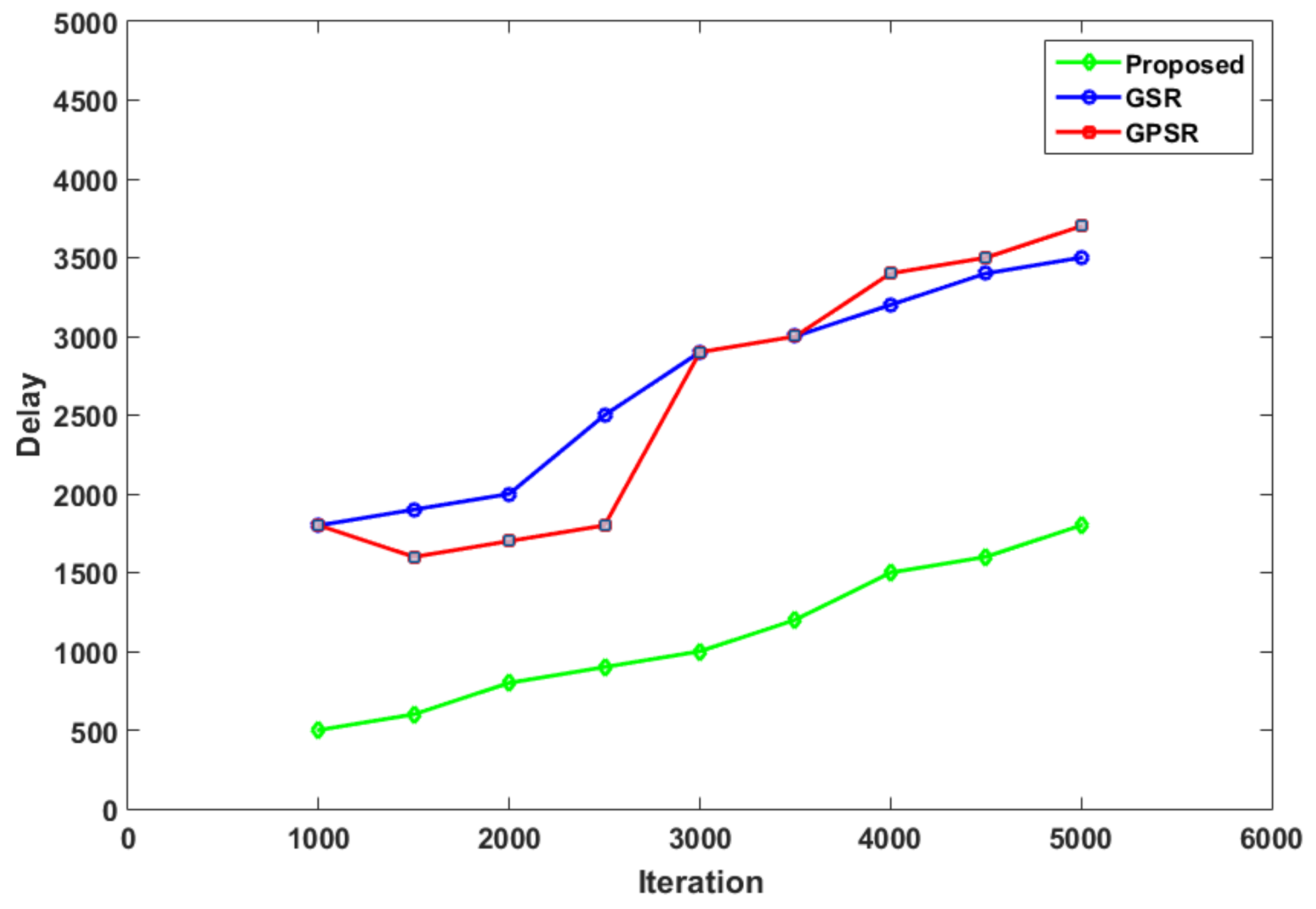

Figure 11

Comparative Analysis of Delay 


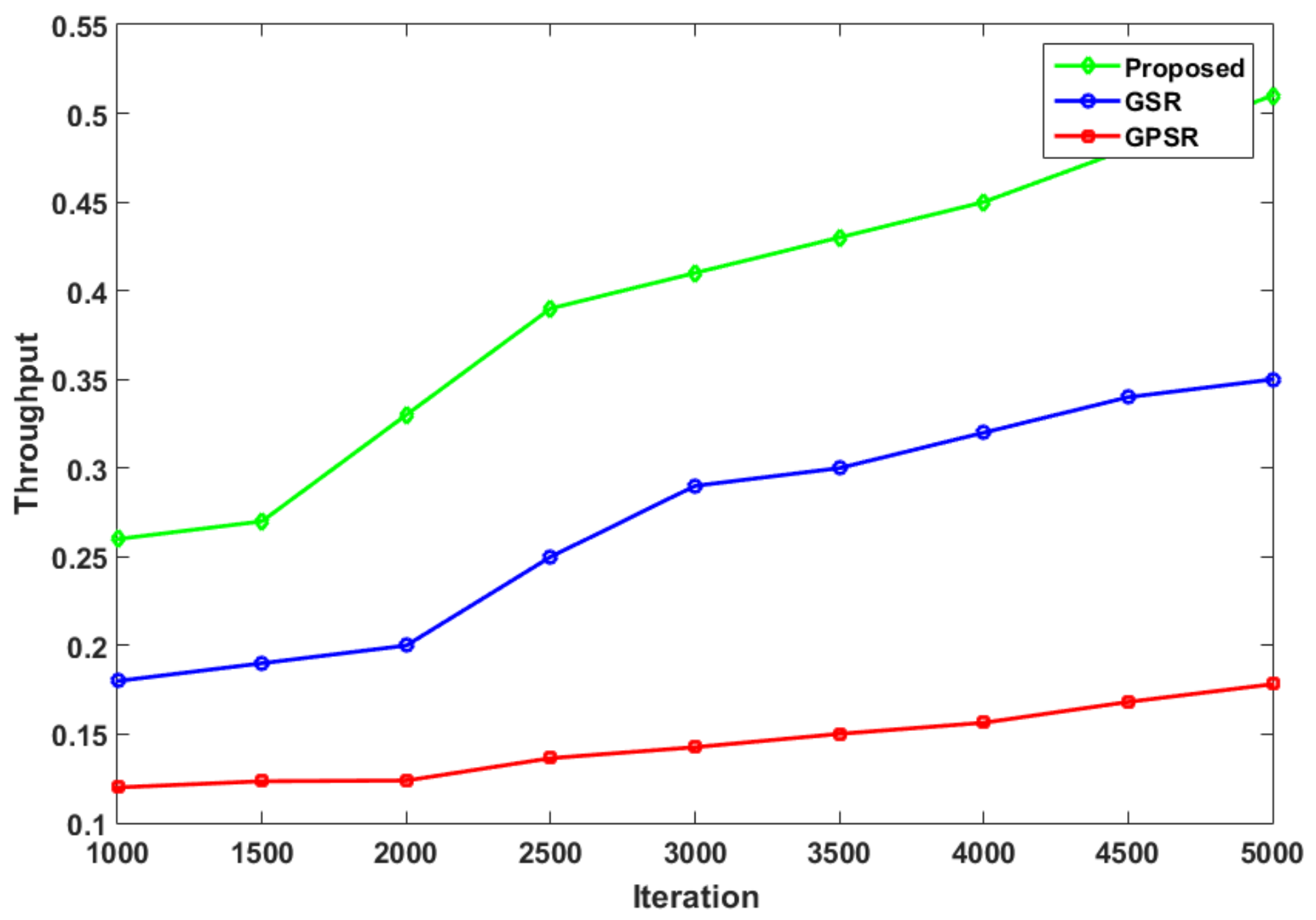

Figure 12

Comparative Analysis of Throughput 